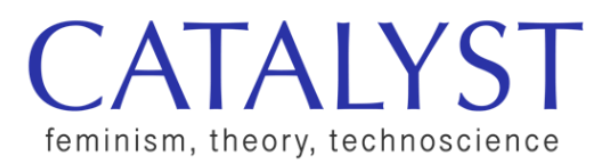

\title{
Book Review | Cooking Data: Culture and Politics in an African Research World, by Crystal (Cal) Biruk (Duke University Press, 2018)
}

\author{
Angela Okune \\ University of California, Irvine \\ aokune@uci.edu
}

An ethnography of survey research in Malawi, Cooking Data traces the social lives of numbers, demonstrating how the making of quantitative global health data requires a sociotechnical knowledge infrastructure to manage the people, places, and things that characterize the messiness of fieldwork. The work describes demographers' efforts at standardization and "quality control" that are established to achieve a goal of "clean" data. But in exploring the everyday relations between persons, data, technologies, and infrastructure, the book reveals that the making of such "good" data requires creativity and tinkering as much as harmonization and consistency. In other words, the book demonstrates how all data is in fact "cooked" or produced by unique processes and practices of production. The book's middle three chapters in particular foreground the expertise and importance of fieldworkers who are key to ensuring that data collection proceeds smoothly and that produced data is deemed objective, clean, and consumable, as determined by survey research standards.

Such data standards make possible the stable and fixed numerical representation of everyday sociocultural entanglements. Building on work from science and technology studies that has revealed the ways in which classification systems and standards are politically and ethically charged, Biruk complicates the assumed binary categories of "raw" and "cooked" data, illustrating the co-production of

Okune, Angela (2020). Review of Cooking Data: Culture and Politics in and African Research World, by Crystal Biruk (Duke University Press, 2018). Catalyst: Feminism, Theory, Technoscience, 6(2), pp. $1-3$.

http://www.catalystjournal.org | ISSN: 2380-3312

(c) Angela Okune, 2020 | Licensed to the Catalyst Project under a Creative Commons Attribution Non-Commercial No Derivatives license 
quantitative health data and their social worlds. For example, two extended vignettes in Chapter 5 demonstrate how even after the collection of boxes upon boxes of data (evinced by the powerful cover image), the numbers underlying "evidence-based" claims in policy research are never stable. Despite being considered the final representative form of knowledge, Biruk demonstrates how survey numbers continue to undergo transformation as they travel between actors with different goals across multiple scales of the research assemblage.

The continued relevance of Biruk's work is clear in the midst of the global COVID19 pandemic. Some local and national leaders have espoused a "data-driven" approach to reopening their economies, while others have leveraged a lack of data to downplay the significance and severity of the pandemic. Such examples reinforce Biruk's point that the epistemological rigor of numbers does not in fact determine whether they become mobilized (or ignored) as "evidence." Rather, evidence is "as cooked in its sites of consumption as it is in in its sites of production" (p. 199). At a time when trust in numbers is increasingly shaken, Biruk offers nuanced insights on the production of numbers that prompts discussion about the role of feminist science studies to both critically examine how numbers attain their authority and simultaneously build capacity for fact-based decision making in a post-truth era where powerful leaders intentionally spread harmful misinformation. Biruk explores this tension between "being useful" and "being critical" in the book's conclusion, suggesting that the enduring potential of an ethnographic mode of critique lies in the figure of the fieldworker "betwixt and between" (p. 210) who, well aware of the ambiguities and blind spots on which dichotomies are built, navigates them carefully but without resolution.

Reflecting importantly on the non-innocence of the discipline of anthropology and their own complicity within the research apparatus, Biruk asks anthropologists of science to pay attention to how our own data is also cooked, encouraging scholars to cultivate an awareness of the kinds of evidence we too often take for granted: "How might we provoke ourselves to imagine ways of being anthropological that are not governed quite so much by either the compulsion to critique and/or to be useful in particular ways?" (p. 213). Beyond advising how to "make global projects work better" (p. 201), social scientists of science are well positioned to practice and experiment on how we might begin to do "data" differently ourselves. Given that, as Biruk points out, anthropologists "take their time, celebrate messy or dirty data, and see questions not as conclusively answerable...but as provocations of new questions" (p. 203), how might we begin to model, experiment, and cook our own data differently? 
In a Somatosphere Book Forum interview, Biruk explains that "ethnography is itself a practice of care that can model how obligation, fragile solidarities, and complicities are enabling as much as constraining" (Tichenor, Mika, \& Droney, 2019, p. 18). Building on this formulation of ethnography, can we practice caring for our own ethnographic data and research relationships in such a way as to "reshuffle and undo the fixity of taken for granted concepts [like data] that structure global health worlds" (Tichenor, Mika, \& Droney, 2019, p. 18)? The moment is ripe for disciplines like anthropology whose data is often considered "clunky [and] complex" (Biruk, 2018, p. 213) to study and develop sociotechnical data systems that push our discipline and others in new directions, not in search of the "best solution," which we know is a futile aim, but towards decolonizing existing global knowledge systems and engendering new forms of research relationships.

\section{Reference}

Tichenor, M., Mika, M., \& Droney, D. (2019). Book forum: Crystal (Cal) Biruk's Cooking Data, Ramah McKay's Medicine in the Meantime, and Noémi Tousignant's Edges of Exposure. Somatosphere. http://somatosphere.net/2019/book-forumcooking-data-medicine-in-the-meantime-edges-of-exposure.html/

\section{Author Bio}

Angela Okune is a doctoral candidate in the Anthropology Department at the University of California, Irvine. She studies data practices and infrastructures of research groups working in and on Nairobi, Kenya, to explore broader questions of equity, knowledge production, and socioeconomic development in Africa. 